\title{
Risk Factor Analysis of Postoperative Pancreatic Fistula After Distal Pancreatectomy, with A Focus on Pancreas-Visceral Fat CT Value Ratio and Serrated Pancreatic Contour, Evaluated Using Preoperative CT Images: A Retrospective Single Center Study
}

\section{Koki Maeda}

Mie University Graduate School of Medicine

Naohisa Kuriyama ( $\nabla$ naokun@med.mie-u.ac.jp )

Mie University Graduate School of Medicine

Takuya Yuge

Mie University Graduate School of Medicine

Takahiro Ito

Mie University Graduate School of Medicine

Kazuyuki Gyoten

Mie University Graduate School of Medicine

\section{Aoi Hayasaki}

Mie University Graduate School of Medicine

\section{Takehiro Fujii}

Mie University Graduate School of Medicine

Yusuke lizawa

Mie University Graduate School of Medicine

Yasuhiro Murata

Mie University Graduate School of Medicine

Akihiro Tanemura

Mie University Graduate School of Medicine

Masashi Kishiwada

Mie University Graduate School of Medicine

Hiroyuki Sakurai

Mie University Graduate School of Medicine

Shugo Mizuno

Mie University Graduate School of Medicine 


\section{Research Article}

Keywords: distal pancreatectomy, postoperative pancreatic fistula, CT value, serrated pancreas, visceral fat, diabetes mellitus

Posted Date: March 3rd, 2022

DOI: https://doi.org/10.21203/rs.3.rs-1377078/v1

License: (1) This work is licensed under a Creative Commons Attribution 4.0 International License.

Read Full License 


\section{Abstract}

Background: In pancreaticoduodenectomy, the pancreas-visceral fat CT value ratio and serrated pancreatic contour on preoperative CT have been revealed as risk factors for postoperative pancreatic fistulas. We aimed to evaluate whether they could also serve as risk factors for postoperative pancreatic fistulas after distal pancreatectomy.

Methods: A total of 251 patients that underwent distal pancreatectomy at our department from 2006 to 2020 were enrolled for the study. We retrospectively analyzed risk factors for postoperative pancreatic fistulas after distal pancreatectomy using various pre and intraoperative factors, including preoperative CT findings, such as pancreas-visceral fat CT value ratio and serrated pancreatic contour.

Results: The study population included 147 male and 104 female participants (median age, 68 years; median body mass index, $21.4 \mathrm{~kg} / \mathrm{m} 2)$, including 64 patients with diabetes mellitus (25.5\%). Preoperative CT evaluation showed a serrated pancreatic contour in 80 patients $(31.9 \%)$, a pancreatic thickness of 9.3 $\mathrm{mm}(4.0-22.0 \mathrm{~mm})$, pancreatic parenchymal CT value of $41.8 \mathrm{HU}(4.3-22.0 \mathrm{HU})$, and pancreas-visceral fat CT value ratio of -0.41 (-4.88 to -0.04 ). Postoperative pancreatic fistulas were developed in $34.2 \%$ of the patients. Univariate analysis of risk factors for postoperative pancreatic fistulas showed that younger age $(P=0.005)$, high body mass index $(P=0.001)$, absence of diabetes mellitus $(P=0.002)$, high preoperative $C$-reactive protein level $(P=0.024)$, pancreatic thickness $(P<0.001)$, and high pancreatic parenchymal CT value $(P=0.018)$ were significant risk factors; however, pancreas-visceral fat CT value ratio $(P=0.337)$ and a serrated pancreatic contour $(P=0.122)$ did not serve as risk factors. Multivariate analysis showed that high body mass index $(P=0.032)$, absence of diabetes mellitus $(P=0.001)$, and pancreatic thickness $(P<0.001)$ were independent risk factors.

Conclusion: The pancreas-visceral fat CT value ratio and serrated pancreatic contour evaluated using preoperative CT were not risk factors for postoperative pancreatic fistulas after distal pancreatectomy. High body mass index, absence of diabetes mellitus, and pancreatic thickness were independent risk factors, and a close-to-normal pancreas with minimal fat deposition or atrophy is thought to indicate a higher risk of postoperative pancreatic fistulas after distal pancreatectomy.

\section{Background}

There is a higher incidence of postoperative pancreatic fistula (POPF) after distal pancreatectomy (DP) than thatafter pancreaticoduodenectomy (PD) [1];POPFisthe most clinically problematic complication of these procedures. Various risk factors for POPF after DP have been reportedthus far, including obesity, younger age, malnutrition, pancreatic thickness, and soft pancreatic texture (i.e.,soft pancreas)[2-5]. Althoughmost of these factors can beevaluated preoperatively, pancreatictexturecan be determined only by intraoperative findings. Recently,fat depositioninpancreaticparenchyma-related to soft pancreas-has been reported tobe strongly associated with POPF after PD, and it is represented by alow pancreatic parenchymal CT value $[6,7]$. In this regard, we have recently reportedthat the pancreas-visceral fat CT 
value ratio (PVFR) and serrated pancreatic contour,which can be obtained by preoperative CT images,were associated with fat deposition of the pancreatic parenchyma, and thatthese factors were selected as strong risk factors of POPF after PD [8]. However, these factors have not been examined for patients that underwent DP.

Thus, the aim of this study was to elucidate the risk factors for POPF after DP and to verify whether PVFR and serrated pancreatic contour could also serve asrisk factors for POPF after DP, as they didfor POPF after PD.

\section{Methods}

\section{Patients}

Of the 259 patients who underwent DP atthe Department of Hepatobiliary Pancreatic and Transplant Surgery of Mie University Hospital during the 15-year period from January 2006 to December 2020, we excluded three patients whose preoperative CT images could not be referenced and five patients whosepancreatic parenchyma evaluations were difficult (two patients with remnant pancreatic duct stent after pancreaticoduodenectomy, two patients with severe atrophy/calcification, and one patient with pancreatic blastoma). The remaining 251 patients were identified as the study population.

Theprotocol for this research was approved by a suitably constituted Ethics Committee at the institution(Committee of the Institutional Review Board at Mie University of Japan, Approval No.H2021024), and the study conformed to the provisions of the Declaration of Helsinki. Informed consents were obtained from all the participants through an opt-out form. Participants were explained that they could opt out of participation by filling out an opt-out form. This consent procedure was approved by the Committee of the Institutional Review Board at Mie University of Japan. The study received ethical approval for the anonymization of patient data, the absence of risks to the patient, and the potential benefit for the adequate management of POPF based on unbiased information. All data were fully anonymized beforewe accessed them.

Risk factor analysis for postoperative pancreatic fistula

Perioperative information and CT images were retrospectively extracted from medical records; univariate and multivariate analyses of risk factors for POPF after DP were performed.Preoperative factors that were evaluated in this studyincluded age, sex, body mass index (BMI), history of preoperative diabetes mellitus (DM), diagnosis, history of preoperative chemoradiotherapy, and the hematologic examinations. Additionally, the preoperative nutritional scores evaluated included the prognostic nutritional index (PNI), neutrophil-to-lymphocyte ratio (NLR), and platelet-to-neutrophil ratio (PNR).

Intraoperative factors that were evaluated included operation time, intraoperative blood loss, surgical procedure (laparotomy or laparoscopy), combined splenectomy, combined portal vein resection, combined celiac axisresection, and simultaneous resection of the alimentary tract. Pancreatictexture 
could not be confirmed by laparoscopic surgery, andthe descriptionof pancreatic texture was omitted in the surgical recordsin some open surgery cases,therefore,this factorwas not evaluated in the presentstudy.

POPFwas defined and graded according to the International Study Group on Pancreatic Surgery (ISGPS) in 2016 [9], with Grades B and C set as clinicallyrelevant POPF (CR-POPF). The Clavien-Dindo classification (CD classification) was used for severity classification of postoperative complications [10].

Evaluation of pancreatic fat deposition and pancreatic morphology onpreoperative CT

To represent pancreatic fat deposition,the PVFR was determined from preoperative CT byusing the method described in our previous report [8]. The CT values were measured at thefuture remnant pancreatichead for the pancreatic parenchyma and the left side of the stomach for visceral fat (Figure 1). The CT values (mean values) were measured at four different locations with regions of interest (ROIs) of $15-30 \mathrm{~mm}^{2}$ at sites where the vessels did not overlap while comparing the non-contrast CT with the contrast-enhanced CT; the mean CT values of the four measurementswas calculated and adopted. The PVFR was calculated as the mean pancreatic parenchymal CT value / mean visceral fat CT value.

Pancreatic morphology was categorized as normal with a smooth margin (smooth type) oran irregular serrated pancreatic contour with protrusionsof $3 \mathrm{~mm}$ or more (serrated type) (Figure 2). The pancreatic parenchymal thickness was the value in the direction perpendicular to the long axis of the pancreas on the pancreatic dissection line in the horizontal section of the preoperative $\mathrm{CT}$, identified prior to postoperative CT.

Surgical procedure

Pancreatic stump closure for open surgery was performedusing hand-sewn or stapled closure technique. In the case of hand-sewn occlusion of the pancreatic cut end, the pancreatic parenchyma was transected using anultrasonic coagulation cuttingdevice (SonoSurg; Olympus Optical Co. Ltd., Tokyo, Japan) followed by either main pancreatic duct ligation and suture ligation using the fish-mouth method. Regarding the staple closure technique,pancreatic parenchyma was divided witha bare or meshreinforced triple-row stapler (Endo GIA Tri-Staple ${ }^{\mathrm{TM}}$ or NEOVEIL Endo GIA Reinforced Reload,Covidien, North Haven, CT, USA) using a purple $(3 \mathrm{~mm})$ or black $(4 \mathrm{~mm})$ cartridge, selected by the surgeon according to the thickness of the transection line. In laparoscopic surgery, pancreatic stump was closed usinga linear stapler or endoscopic hand-sewn technique as well as open surgery. None of the cases involved gastrointestinal anastomosis with the remnant pancreas. A closed suctiondrain was placed at the pancreatic stump and/or left subphrenic spacebased on the surgeon' judgement.

Statistical analysis

The normality of data distribution was investigated using the Shapiro-Wilk test; parametric continuous variables were analyzed using Student's t-test, and the non-parametric continuous variables were 
analyzed using a Mann-Whitney $U$ test. Categorical variables were analyzed using a $\chi^{2}$ test. The significance level was set to $P<0.05$; multivariate analysis was conducted using multiple logistic analysis, with variables showing significant differences in univariate analysis set as independent variables. The optimal cut-off value was determined using the Youden index from the receiver operating characteristic (ROC) curve. All statistical analyses were conducted using SPSS (version 26; IBM Japan, Tokyo Japan).

\section{Results}

Characteristics of the patients and preoperative CT evaluation

Table 1 presentsthe data for patient characteristics and intraoperative factors. The median patient age was 68 years (3-89 years), and the study population included147 male (58.7\%) and 104 female (41.3\%) patients. The median BMI was $21.4 \mathrm{~kg} / \mathrm{m}^{2}\left(13.6-34.7 \mathrm{~kg} / \mathrm{m}^{2}\right)$, and 64 patients hadpreoperative DM (25.5\%); the most common diagnosis waspancreatic ductal adenocarcinoma (PDAC; 105 cases, 41.8\%). Preoperative therapy was performed in 67 patients (26.7\%): chemoradiotherapy wasperformed in 54 patients and chemotherapy in 13 patients. The median operation time was 322 minutes (132-830 minutes), and the median blood loss was $404 \mathrm{~mL}(0-5,033 \mathrm{~mL})$. Laparoscopic surgery was performed in 87 patients (34.7\%).

Preoperative CT evaluation revealed a serrated type pancreas in 80 patients (31.9\%) (Table 2). The median pancreatic thickness was $9.3 \mathrm{~mm}(4.0-22.0 \mathrm{~mm})$, median CT value of the pancreatic parenchyma was $41.8 \mathrm{HU}(4.3-73.2 \mathrm{HU})$, and median PVFR value was -0.41 (-4.88 to -0.04$)$.

Incidence and severity of postoperative pancreatic fistula

CR-POPFdeveloped in86 patients (34.3\%), including Grade Bin 83 patients (33.1\%) and Grade Cin three patients (1.2\%) (Table 3 ). Among the patients withGrade B, 30 improved with therapeutic antibiotics alone (CD classification II), while53 required drain tube replacement and/or additional interventions (CD classification IIla). Grade Cwas observed in three patients, of whichone patient underwent additional resection of the residual pancreas at the splenic hilum using the Warshaw technique (CD classification IIIb), oneunderwent atransverse colon resection and splenic artery stent placement due to puncture of the splenic artery pseudoaneurysm (CD classification IVa), and one developed septic shock and required intensive care in the intensive care unit (CD classification IVb).

Risk factors of clinically relevant postoperative pancreatic fistula

Univariate analysis of the risk factors for CR-POPF identified younger age $(P=0.005)$, high BMI $(P=$ $0.001)$, absence of DM $(P=0.002)$, high preoperative $C R P$ value $(P=0.024)$, pancreatic thickness $(P<$ $0.001)$, and high pancreatic parenchymal CT value $(P=0.018)$ as risk factors (Table 4). However, a serrated type pancreas $(P=0.122)$ and PVFR $(P=0.373)$ were not identified asrisk factors. The durationof hospital stay was significantly longerin the CR-POPF group $(P<0.001)$. 
Multivariate analysis revealed that high BMI (odds ratio: 1.111; 95\% Cl: 1.709-1.223; P = 0.032), absence of DM (odds ratio: -1.330; 95\% Cl: 0.123-0.567; P =0.001), and pancreatic thickness (odds ratio: 1.220; 95\% Cl: 1.092-1.363; P < 0.001) were independent risk factors for CR-POPF (Table 4). The optimal cut-off values by ROC analysis were $22.4 \mathrm{~kg} / \mathrm{m}^{2}$ (AUC: 0.632 ; sensitivity: 0.581 ; specificity: 0.697 ) for $\mathrm{BMI}$ and $12.4 \mathrm{~mm}$ (AUC: 0.662; sensitivity: 0.372; specificity: 0.927) for pancreatic thickness (Figure 3 ).

\section{Discussion}

The incidence of POPF after DP differs between reports, but the results of a large-scale, multi-center, joint study in Japan in 2015[11] reported an incidence of $28.2 \%$ with open surgery and $18.4 \%$ withlaparoscopic surgery. Several DP-related randomized controlled trials after 2016 [12-14]reported POPF incidence rates of $16.0 \%-18.9 \%$, and a recent meta-analysis [15] reported an incidence of $20.4 \%$, indicating thatthis complication hasa relatively high frequency of occurrence regardless of advancementsin surgical techniques. The incidence of CR-POPF in the present study was 34.3\%, higherthan that in previous reports. In the present study, the incidence of CR-POPF did not differ significantly according to pancreatic stump closure method (hand-sewn closure vs. stapler). Furthermore, thehand-sewn closure method in the laparoscopic surgery-previously adopted in our department-wasperformed for 33 patients, but CR-POPF was observed inonly 10 cases (30.3\%);thus, incidence of CR-POPF in our departmentwas not particularly high. Although various reports have evaluated the optimal pancreatic stump closure method for POPF reduction, no consensus has been established, andfurther studiesare needed to identifyaclosure method that reduces the incidence of CR-POPF. Furthermore, based on our previous report[16], aconsiderationfrom the perspectives of intraoperative and postoperative management indicated that the inappropriate drainage tube placement and/or number of drain tubes in our department may have increased the number of cases requiring additional intervention and may have also resulted in excessive use of postoperative therapeutic antibiotics. If the drainage is appropriate, the drain can be removed at an early stage; furthermore, if the indication for therapeutic antibiotic use is judged more strictly, then it would not result in Grade BCR-POPF, and there may be more cases that would heal with a biochemical leak $(\mathrm{BL})$. Although these are all speculations, further improvements are needed in the future both for intraoperative drain tube placement and the postoperative antibiotic usage method.

The previously reported representative risk factors for POPF after DP include obesity, younger age, malnutrition, and soft pancreas[2-5], but many reports have indicated that pancreatic thickness is the most important risk factor [17]. Univariate analysis in the present study showed that, among the abovementioned factors, high BMI (obesity), younger age, and pancreatic thickness were detected as risk factors, and multivariate analysis showed that high BMI (obesity) and pancreatic thickness were detected as independent risk factors; these results are similar to those in previous reports. The present study also indicated absence of DM as an independent risk factor in multivariate analysis. Although few report have indicatedabsence of DM as a risk factor, a recent meta-analysis [15] reported that DM was a significant protective factor for CR-POPF after DP. Exocrine function as well as endocrine function was reduced in DM patients[18], therefore this may have increased the incidence of POPF. 
Several reports have performedrisk factor analysis of POPF after pancreatic resection by using preoperative CT values, aswe have focused on in the present study. In particular, a frequently used value is the pancreas/spleen $\mathrm{CT}$ value ratio ( $\mathrm{P} / \mathrm{S}$ ratio), obtained by dividing the pancreatic parenchymal $\mathrm{CT}$ value by the spleen $\mathrm{CT}$ value;it is basedon the liver/spleen $\mathrm{CT}$ value ratio commonly used for evaluating fatty liver [19]. The P/S ratio has been reported to represent pancreatic fat deposition and is an index of a soft pancreas in both patients who underwent PD [6] and DP [20], and a significant risk factor of POPF. Furthermore, we had previously discovered that the PVFR, which is basedon the P/S ratio and serrated pancreaticcontour could be used to predict the fat deposition of the pancreatic parenchyma, and that thesefindings are risk factors of POPF in PD [8]. Therefore, in the present study, we calculated the PVFR and identifiedthe serrated type pancreas to verify if theycould also serveas risk factors of POPF after DP. Specifically, we initially hypothesized that the PVFRand serrated type pancreas, which represent pancreatic fat deposition and a soft pancreas, reflect the fragility of the pancreas, and that a fragile pancreatic stump would be prone to collapse and be more likely to induce POPF. However, our univariate and multivariate analysis showed that thePVFR andserrated type pancreas did not serve as risk factors. Rather, the pancreatic parenchymal CT value was significantly higher in the CR-POPF group, indicating that pancreas fat depositionin this group might be milder than in the non POPF/BL group. Furthermore, an investigation on POPF after DP by Mori et al. [7]indicated that patients with a high pancreatic parenchymal CT value or $\mathrm{P} / \mathrm{S}$ ratio had mild pancreatic fat deposition and maintained pancreatic exocrine function, and showeda significantly higher POPF incidence than those who do not; these results are similar to the findingsof the present study. It is clear that a soft pancreas is a risk factor for POPF after PD, but whether it is a risk factor for POPF after DP, is still controversial [8, 20-22]. However, the present results seem to indicate that the risk of POPF after DPis higher among patients with less pancreas fat deposition, maintained pancreatic endocrine/exocrine function, less atrophy, and thickerpancreas compared to those with a fragile pancreas with fat deposition. The present results contradict our hypothesis, but to our knowledge, there have been no previous studies that investigated PVFR and morphological characteristics of the pancreas (i.e., serrated type pancreas) as risk factors of POPF after DP,therefore, the present study can provide valuableinvestigative data in this regard.

The following aspects can be consideredas limitations of the present study: (i) this wasa retrospective study with a small sample size froma single facility; (ii) the study period was long,potentially introducing various biases relatedto changes in postoperative management policies, surgical techniques, and pancreatic stump closure method; (iii) the evaluation of pancreatic morphology and CT value measurement method lacked objectivity; although criteria have been set for evaluation of pancreatic morphology, the influence of the evaluator's subjectivity cannot be excluded;(iv) furthermore, CT value measurements were conducted so as not to overlap with the blood vessels and ducts in comparisonwith contrast-enhanced CT, but this process was not conducted mechanically, and there were problems with its accuracy. The conclusions drawn from the results of the present study should be interpreted in the light of these limitations andevaluated further in future studies.

\section{Conclusion}


PVFR and serrated pancreatic contour evaluated with preoperative CT did not constitute risk factors of POPF after DP, unlike the findings for PD. High BMI, absence of DM, and pancreatic thickness have been shown to be independent risk factors, and the risk of POPF after DP is thought to be higher among those with a closer-to-normal pancreas with minimal fat deposition or atrophy.

\section{Abbreviations}

BL Biochemical leak

BMI Body mass index

CR clinically relevant

CRP C-reactive protein

DM Diabetes mellitus

DP Distal pancreatectomy

ISGPS International Study Group on Pancreatic Surgery

NLR Neutrophil-to-lymphocyte ratio

PD Pancreaticoduodenectomy

PNI Prognostic nutritional index

PNR Platelet-to-neutrophil ratio

POPF Postoperative pancreatic fistulas

PVFR Pancreas-to-visceral fat ratio

ROC Receiver operating characteristic

ROI Regions of interest

\section{Declarations}

Ethics approval and consent to participate:

Theprotocol for this research was approved by a suitably constituted Ethics Committee at the institution(Committee of the Institutional Review Board at Mie University of Japan, Approval No.H2021024), and the study conformed to the provisions of the Declaration of Helsinki. Informed consents were obtained from all the participants through an opt-out form. Participants were explained that they could 
opt out of participation by filling out an opt-out form. This consent procedure was approved by the Committee of the Institutional Review Board at Mie University of Japan. The study received ethical approval for the anonymization of patient data, the absence of risks to the patient, and the potential benefit for the adequate management of POPF based on unbiased information. All data were fully anonymized beforewe accessed them.

Consent for publication:

Not applicable

Availability of data and materials:

The datasets generated and/or analyzed during the current study are not publicly available asthey consist of confidentialpatient data;however,data will be made available from the corresponding author on reasonable request.

Competing interests:

The authors declare that they have no competing interests

Funding:

Not applicable

Author's contribution:

Study design: MK and KN. Data analysis: MK. Data acquisition: YT, IT, GK, HA, FT, IY, MY, TA, KM and SH.Draft and revision of the manuscript: MK, KN. Critical revision and accountability of the study: MS. All authors read and approved the final manuscript.

Acknowledgements:

We would like to thank Editage (www.editage.com) for English language editing.

\section{References}

1. McMillan MT, Christein JD, Callery MP, Behrman SW, Drebin JA, Hollis RH, et al. Comparing the burden of pancreatic fistulas after pancreatoduodenectomy and distal pancreatectomy. Surgery. 2016;159:1013-22.

2. Zhou Y, Drake J, Deneve JL, Behrman SW, Dickson PV, Shibata D, et al. Rising BMI is associated with increased rate of clinically relevant pancreatic fistula after distal pancreatectomy for pancreatic adenocarcinoma. Am Surg. 2019;85:1376-80.

3. Ecker BL, McMillan MT, Allegrini V, Bassi C, Beane JD, BeckmanRM, et al. Risk factors and mitigation strategies for pancreatic fistula after distal pancreatectomy: analysis of 2026 resections from the 
International, Multi-institutional Distal Pancreatectomy Study Group. Ann Surg. 2019;269:143-9.

4. Kawaida H, Kono H, Watanabe M, Hosomura N, Amemiya H, Fujii H. Risk factors of postoperative pancreatic fistula after distal pancreatectomy using a triple-row stapler. Surg Today. 2018;48:95-100.

5. Kollár D, Huszár T, Pohárnok Z, Cselovszky É, Olah A. A review of techniques for closure of the pancreatic remnant following distal pancreatectomy. Dig Surg. 2016;33:320-8.

6. Yardimci S, Kara YB, Tuney D, Attaallah W, Ugurlu MU, Dulundu E, et al. A simple method to evaluate whether pancreas texture can be used to predict pancreatic fistula risk after pancreatoduodenectomy. J Gastrointest Surg. 2015;19:1625-31.

7. Mori N, Sakurai N, lizawa H.『Predicting postoperative pancreatic fistula after distal pancreatectomy by measuring the CT value ratio of future pancreas remnant to spleen ( $\mathrm{P} / \mathrm{S}$ ratio) in preoperative unenhanced CT images $囚 . J p n$ J Gastroenterol Surg. 2019;52(9):485-93.[Article in Japanese with English abstract].

8. Kusafuka T, Kato H, lizawa Y, Noguchi D, Gyoten K, Hayasaki A, et al. Pancreas-visceral fat CT value ratio and serrated pancreatic contour are strong predictors of postoperative pancreatic fistula after pancreaticojejunostomy. BMC Surg. 2020;20:129.

9. Bassi C, Marchegiani G, Dervenis C, Sarr M, Hilal MA, Adham M, et al. The 2016 update of the International Study Group (ISGPS) definition and grading of postoperative pancreatic fistula: 11 Years after. Surgery. 2017;161:584-91.

10. Dindo D, Demartines N, Clavien PA. Classification of surgical complications: A new proposal with evaluation in a cohort of 6336 patients and results of a survey. Ann Surg. 2004;240:205-13.

11. Nakamura M, Wakabayashi G, Miyasaka Y, Tanaka M, Morikawa T, Unno M, et al. Multicenter comparative study of laparoscopic and open distal pancreatectomy using propensity scorematching. J Hepatobil Pancreat Sci. 2015;22:731-6.

12. Kawai M, Hirono S, Okada K, Sho M, Nakajima Y, Eguchi H, et al. Randomized controlled trial of pancreaticojejunostomy versus stapler closure of the pancreatic stump during distal pancreatectomy to reduce pancreatic fistula. Ann Surg. 2016;264:180-7.

13. Uemura K, Satoi S, Motoi F, Kwon M, Unno M, Murakami Y. Randomized clinical trial of duct-tomucosa pancreaticogastrostomy versus handsewn closure after distal pancreatectomy. Br J Surg. 2017;104:536-43.

14. Wennerblom J, Ateeb Z, Jönsson C, Björnsson B, Tingstedt B, Williamsson C, et al. Reinforced versus standard stapler transection on postoperative pancreatic fistula in distal pancreatectomy: multicentre randomized clinical trial. Br J Surg. 2021;108:265-70.

15. Chong E, Ratnayake B, Lee S, French JJ, Wilson C, Roberts KJ, et al. Systematic review and metaanalysis of risk factors of postoperative pancreatic fistula after distal pancreatectomy in the era of 2016 International Study Group pancreatic fistula definition. HPB (Oxford). 2021;23:1139-51.

16. Maeda K, Kuriyama N, Nakagawa Y, Ito T, Hayasaki A, Gyoten K, et al. Optimal management of peripancreatic fluid collection with postoperative pancreatic fistula after distal pancreatectomy: 
Significance of computed tomography values for predicting fluid infection. PLoS one 2021;16:e0259701.

17. Miyasaka Y, Mori Y, Nakata K, Ohtsuka T, Nakamura M. Attempts to prevent postoperative pancreatic fistula after distal pancreatectomy. Surg Today 2017;47:416-24.

18. Saisho Y. Pancreas volume and fat deposition in diabetes and normal physiology: Consideration of the interplay between endocrine and exocrine pancreas. Rev Diabet Stud. 2016;13:132-47.

19. Piekarski J, Goldberg HI, Royal SA, Axel L, Moss AA. Difference between liver and spleen CT numbers in the normal adult: Its usefulness in predicting the presence of diffuse liver disease. Radiology. 1980;137:727-9.

20. Fukuda Y, Yamada D, Eguchi H, Iwagami Y, Noda T, Asaoka T, et al. A novel preoperative predictor of pancreatic fistula using computed tomography after distal pancreatectomy with staple closure. Surg Today. 2017;47:1180-7.

21. Ridolfini MP, Alfieri S, Gourgiotis S, Di Miceli D, Rotondi F, Quero G, et al. Risk factors associated with pancreatic fistula after distal pancreatectomy, which technique of pancreatic stump closure is more beneficial? World J Gastroenterol. 2007;13:5096-100.

22. Kah Heng CA, Salleh I, San TS, Ying F, Su-Ming T. Pancreatic fistula after distal pancreatectomy: incidence, risk factors and management. ANZ J Surg. 2010;80:619-23.

\section{Tables}

Tables are available in the Supplementary Files Section.

\section{Figures}



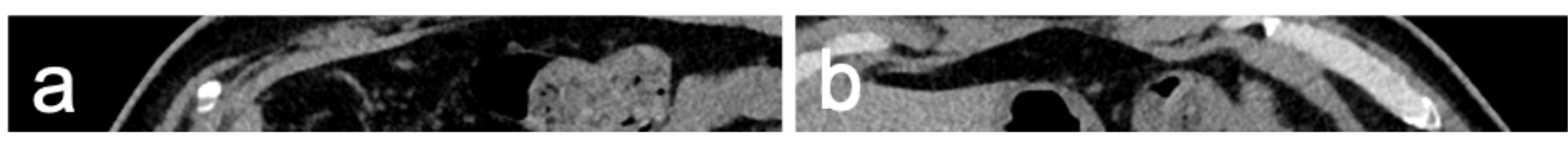

Figure 1

Measurement of the CT value of the remnant pancreatic parenchyma (a) and visceral fat (b).

HU: Hounsfield units, PVFR: pancreas-visceral fat ratio

\section{Smooth type \\ Serrated type}

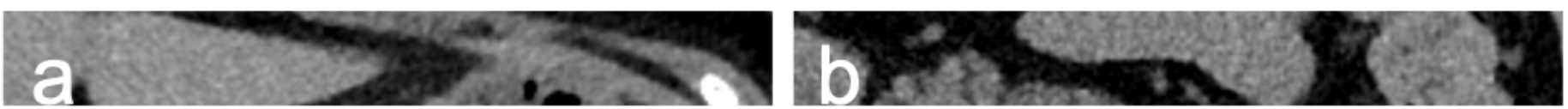

Figure 2

Classification of pancreatic morphology

a) Smooth type: smooth interlobular border 
b) Serrated type: feathery, irregular interlobular border, and a protrusion of more than $3 \mathrm{~mm}$.

\section{Figure 3}

ROC analysis of therisk factors for POPF after DP

ROC: receiver operating characteristic, BMI: body mass index AUC: area under the curve, PPV: positive predictive value, NPV: negative predictive value, POPF: postoperative pancreatic fistula,DP: distal pancreatectomy

\section{Supplementary Files}

This is a list of supplementary files associated with this preprint. Click to download.

- Table1.xlsx

- Table2.xlsx

- Table3.xlsx

- Table4.xlsx 